\title{
Associação entre eventos estressores e citocinas inflamatórias e anti- inflamatórias em pessoas idosas longevas
}

\author{
Association between stressor events and inflammatory and anti-inflammatory cytokines in \\ long-lived older people
}

\author{
Ingridy Fátima Alves Rodrigues' (D) \\ Vicente Paulo Alves' $\mathbb{D}$ \\ Lucy de Oliveira Gomes' (ID \\ Daniele Sirineu Pereira ${ }^{2}$ (D) \\ Otávio de Toledo Nóbrega ${ }^{3}$ (I) \\ Karla Helena Coelho Vilaça e Silva' (D)
}

\section{Resumo}

Objetivo: investigar a associação entre a frequência de eventos estressores e citocinas em pessoas idosas longevas. Métodos: os participantes responderam a um questionário constituído de variáveis sociodemográficas, indicaram quais eventos estressores constantes no Inventário de Eventos Estressores de vida ocorreram nos últimos cinco anos e responderam a escala de depressão geriátrica (GDS). Foram dosados por citometria de fluxo: interleucina (IL) 10, IL-6, IL-4, IL-2, fator de necrose tumoral (TNF- $\alpha$ ) e interferon gama (IFN- $\gamma$ ). A análise descritiva foi realizada para a caracterização da amostra. Para investigar a associação entre as variáveis foi desenvolvido um modelo de regressão linear múltipla, utilizando o método Backward. Resultados: Participaram da pesquisa 91 pessoas idosas com média de idade de 82 anos. Mais da metade da amostra relatou morte de ente querido como o evento estressor mais prevalente (61\%). Nessa amostra foi possível perceber que quanto mais eventos estressores foram relatados, menor o nível de IL-4 ( $p=0,046$ ), da mesma forma que o estado civil viuvez, onde os dados mostraram que quem é viúvo tem menos eventos estressores em comparação a quem é casado $(p=0,037)$. Conclusão: Evidenciou-se a importância de um olhar mais cuidadoso dos profissionais de saúde na avaliação multidimensional da pessoa idosa, de forma que se obtenham subsídios para a implementação de programas e intervenções específicos que possam amenizar a percepção dos eventos estressores vivenciados, colaborando com menores danos decorrentes da imunossenescência.

\footnotetext{
Universidade Católica de Brasília (UCB), Programa de Pós Graduação em Gerontologia. Brasília, DF, Brasil.

2 Universidade Federal de Minas Gerais (UFMG), Programa de Pós-graduação em Ciências da Reabilitação, Escola de Educação Física, Fisioterapia e Terapia Ocupacional. Belo Horizonte, MG, Brasil.

3 Universidade de Brasília (UNB), Faculdade de Medicina. Brasília, DF, Brasil.
}

Financiamento da pesquisa: Programa Nacional de Cooperação Acadêmica (PROCAD). Projeto 88881.068447/2014-01, Convênio CAPES /PROCAD 2972/14. O autor O. T. Nobrega recebeu bolsa de produtividade em pesquisa (Pq2) do CNPq.

Os autores declaram não haver conflito na concepção deste trabalho.

Palavras-chave: Saúde do Idoso. Estresse emocional. Citocina. Interleucina-4. Pessoa Idosa. 


\section{Abstract}

Objective: To investigate the association between the frequency of stressor events and cytokines in long-lived older people. Methods: The participants answered a questionnaire consisting of sociodemographic variables, indicated which stressor events included in the Stressor Life Events Inventory occurred in the last five years and answered the Geriatric Depression Scale (GDS). The following were measured by flow cytometry: interleukin (IL) 10, IL-6, IL-4, IL-2, tumor necrosis factor (TNF- $\alpha$ ) and interferon gamma (IFN- $\gamma$ ). We carried out a descriptive statistical analysis in order to characterize the sample. To investigate the association between the variables, a multiple linear regression model was developed, using the Backward method. Results: 91 older people with an age average of 82 years participated in the research. More than half of the sample reported the death of a loved one as the most prevalent stressor event (61\%). In this sample, it was possible to notice that the more stressor events were reported, the lower the level of IL-4 $(p=0.046)$, as well as the marital status of widowhood, where data showed that those who are widowed have fewer stressor events in comparison to who is married $(p=0.037)$. Conclusion: The importance of a more careful look by health professionals in older people multidimensional assessment was evidenced, so that subsidies are obtained for the implementation of specific programs and interventions that can ease the perception of the stressor events experienced, collaborating with less resulting damage of immunosenescence.

\section{INTRODUÇÃo}

O estresse emocional carrega fortes conotações de quebra de desempenho usual, porém é fator inevitável no curso da vida ${ }^{1}$ e o envelhecimento em si pode ser considerado um fator estressor, pois se relaciona ao aumento de dependência, doenças, perdas de papéis ocupacionais e afetivos ${ }^{2}$. A vivência desses eventos acarreta maior ou menor exigência de recursos emocionais, sociais e intelectuais dependendo do valor que é atribuído a eles ${ }^{3,4}$.

Evento de vida, foi um termo criado por Aldwin 5 e Baltes ${ }^{6}$ para designar uma fase de mudança ocorrida entre períodos relativamente estáveis na vida do indivíduo. Alguns desses eventos são normalmente esperados como a menopausa, entrada na universidade e da aposentadoria, e outros inesperados, como acidentes, desastres naturais e desemprego ${ }^{6}$.

Os eventos inesperados e imprevisíveis, por aumentar a percepção de incontrolabilidade, de ineficácia de enfrentamento ou de ônus excessivo de demandas ${ }^{2}$, apresentam maior potencial para serem vividos como estressantes ${ }^{2,6,7}$. O estresse decorrente desses eventos pode reduzir a capacidade do sistema imunológico para responder a uma injúria ${ }^{8}$. Existem interações entre o Sistema Nervoso Central (SNC), endócrino e imunológico, que são necessárias para que se obtenham respostas adequadas a eventos estressores. Assim, a presença desses eventos pode afetar a circulação e a atividade das células do sistema imunológico?.

Por outro lado, durante o curso de infecções sistêmicas, câncer ou doenças autoimunes, as repercussões imunológicas levam a uma estimulação do SNC que pode levar ao desenvolvimento de sintomas de depressão em indivíduos vulneráveis. A inflamação é, portanto, um importante fator de risco para episódios depressivos maiores, bem como fatores psicossociais mais tradicionais ${ }^{8}$.

O envelhecimento fisiológico está relacionado à alterações do sistema imunológico, o que caracteriza o processo conhecido como imunossenescência. Essas alterações resultam em maior susceptibilidade da pessoa idosa à doenças infecciosas, doenças degenerativas, doenças cardiovasculares, doenças autoimunes e câncer ${ }^{10}$. Dentre as alterações na imunossenescência, destaca-se o aumento de 2 a 4 vezes nos níveis plasmáticos de citocinas tanto pró quanto anti-inflamatórias ${ }^{11}$.

O desequilíbrio na produção e liberação de mediadores inflamatórios caracteriza a inflamação de baixo grau, clinicamente indetectável, denominada 
de inflammaging ${ }^{11,12}$. Esse estado pró-inflamatório ocorre nos idosos mesmo na ausência de doenças associadas e está relacionado com o risco aumentado de doenças crônicas ${ }^{12}$.

Dentre os princípios fundamentais desse paradigma está o aumento de citocinas próinflamatórias e a diminuição de citocinas antiinflamatórias ${ }^{13,14}$. Entre as citocinas pró-inflamatórias estão IL-1, IL-2, IL-6, IL-12, IL-15, IL18, IL-22, IL-23, TNF- $\alpha$, IFN- $\gamma$ e entre as anti-inflamatórias pode-se citar IL-1Ra, IL-4, IL-10, TGF- $\beta^{15}$.

Essas mudanças podem ser analisadas sob uma perspectiva evolutiva e não apenas como prejudiciais ${ }^{16}$. O conhecimento acumulado sugere que, sem a existência da dupla imunossenescência e inflammaging, a longevidade humana seria bastante reduzida o que faz crer que, tentativas de intervenção no sistema imunológico no envelhecimento visando rejuvenescimento devem se basear na manutenção da homeostase geral para melhorar adequadamente as funções imunológicas inflamatórias ${ }^{16}$.

Reconhecer as fontes relacionadas à imunossenescência tem sido base para muitas pesquisas no âmbito do envelhecimento humano, porém a relação entre o impacto do estresse no sistema imune em pessoas idosas, sobretudo aquelas com idade mais avançada ainda são pouco compreendidos. Este estudo teve como objetivo investigar a associação entre a frequência de eventos estressores e citocinas pró e anti-inflamatórias em pessoas idosas longevas.

\section{MÉTODO}

Trata-se de um estudo de corte transversal e quantitativo. A amostra foi obtida por conveniência e recrutada no ambulatório de Geriatria e Medicina Interna do Hospital da Universidade Católica de Brasília (HUCB) entre março de 2016 a maio de 2018. As pessoas idosas que já eram acompanhadas no ambulatório foram convidadas, pessoalmente ou por telefone, a participar da pesquisa de forma voluntária.

Foram realizados treinamentos com a equipe de pesquisadores de campo para padronização das coletas de dados e aplicação dos instrumentos para minimizar os erros de avaliação e problemas no preenchimento do banco de dados.

Como critério de inclusão, optou-se por selecionar aqueles com idade, preferencialmente, maior que 80 anos, não sendo excluídos aqueles que, faltariam menos de um ano e meio para completar a idade pretendida. Assim, foram incluídas pessoas idosas com idade a partir de 78 anos e que eram acompanhadas no ambulatório do HUCB.

O deficit cognitivo foi utilizado como critério de exclusão. Portanto, as 227 pessoas idosas que, inicialmente, consentiram em participar do estudo e assinaram o termo de consentimento, passaram pela triagem cognitiva global, onde foi utilizado o Mini Exame do Estado Mental (MEEM), composto por 30 questões que avaliam a orientação no tempo e espaço, memória episódica, repetição imediata, praxia, funções visuoespaciais e linguagem ${ }^{17}$.

Das 227 pessoas idosas que participaram pela triagem cognitiva global, foram excluídas aquelas que pontuaram abaixo da nota de corte para seu nível de escolaridade, sendo 17 pontos para analfabetos, 22 pontos para idosos com 1 a 4 anos de estudo, 24 pontos para aqueles com 5 a 8 anos de estudo e 26 pontos para os que possuem 9 anos ou mais de estudo, dessa forma, a amostra passou a constar 144 idosos.

Os 144 participantes selecionados responderam a uma entrevista constituída das variáveis sociodemográficas: idade, sexo, cor autodeclarada, se trabalha ou se é aposentado e também sobre a presença de doenças crônicas (cardiopatia, hipertensão arterial, ocorrência acidente vascular encefálico isquêmico, diabetes, câncer, artrite reumatoide, doenças pulmonares, depressão e osteoporose).

Para avaliação da presença de eventos estressores, foi utilizado o Inventário de Eventos Estressantes do Curso de Vida, validado por Aldwin ${ }^{18}$, composto por 31 itens que apresentam eventos estressantes potencialmente vivenciados nos últimos 5 anos anteriores à coleta dos dados. $\mathrm{O}$ instrumento avalia a frequência do acontecimento dos eventos estressantes e o nível de estresse atribuído pelo respondente. As respostas foram agrupadas em 9 categorias: morte de ente querido, doença de ente querido, doença da 
própria pessoa, cuidado de pessoa da família, perda de poder aquisitivo, conflitos familiares, sofrimento de algum tipo de violência, eventos incontroláveis que afetam a descendência, perda de atividade ou amizade apreciada. Os idosos indicaram quais eventos listados estiveram presentes em sua vida nos últimos cinco anos, ou se não aconteceram.

Foi aplicada ainda a escala de depressão geriátrica (GDS), instrumento utilizado para rastrear depressão em pessoas idosas, aqui sendo utilizada a versão curta, composta por 15 perguntas, devendo ser respondidas atribuindo nota 1 para não e 0 para sim, onde o somatório maior ou igual a 5 é indicativo de suspeita de depressão ${ }^{19-21}$.

Para a dosagem de citocinas, a coleta de sangue foi pré-agendada com os participantes para um segundo momento em uma unidade do Laboratório Sabin. Todas as coletas foram realizadas no período da manhã, por profissionais qualificados.

Foi coletada amostra de sangue total de cada participante em tubos com ativador de coagulação e centrifugadas. Essas amostras de sangue total venoso foram transportadas até o Laboratório de Imunogerontologia da $\mathrm{UCB}$ e, no momento da avaliação bioquímica, foram obtidas amostras de soro, ficando mantidas à temperatura de $-80^{\circ} \mathrm{C}$ até serem descongelados para avaliação dos mediadores inflamatórios.

As dosagens das citocinas foram obtidas por citometria de fluxo usando sistema multiplex com um set de imunoensaio baseado em esferas (Human Th1/ Th2/TH17, BD Biosciences, San Diego, Califórnia, USA). Os procedimentos em laboratório seguiram os protocolos fornecidos pelo fabricante do kit. Este permitiu medições para seis mediadores circulantes diferentes: IL-2, IL-4, IL-6, IL-10, IFN- $\gamma$ e TNF- $\alpha$. Os padrões de citocinas liofilizadas e as amostras de soro foram processados juntamente, seguindo protocolo do fabricante, e os resultados obtidos utilizaram o citômetro de fluxo BD FACSCalibur, canal FL4. Os dados foram analisados usando o software FCAP, versão 3.0, (BD Biosciences).

Todas as respostas foram registradas eletronicamente pelo formulário Google e enviados à planilha do banco de dados da pesquisa. Para garantir segurança dos dados contra acesso não autorizado, alteração, divulgação ou destruição não autorizada das informações, os documentos foram criptografados, com acesso restrito e verificação em duas etapas e recurso de navegação segura. Ao final da pesquisa foi realizado um download dos dados para um dispositivo local e apagados os dados da "nuvem".

Dos 144 participantes, 53 foram excluídos por não terem comparecido para a coletada sanguínea para as dosagens das citocinas. A análise final foi realizada com dados referentes a 91 pessoas idosas longevas.

Para verificar a diferença da dosagem dos biomarcadores com o número de eventos estressores de vida foi utilizado o teste Kruskal Wallis.

A associação entre as variáveis categóricas foi verificada por meio do teste qui-quadrado de Person e, quando não foi possível utilizá-lo, foi realizado o teste exato de Fisher.

Ao final, foram selecionadas as variáveis com $p$-valor menor que 0,20 para análise de regressão linear. Em seguida, foi elaborada uma tabela, retirando as variáveis com maiores valores de $p$ até chegar a um modelo com todas as variáveis com $\mathrm{p}$ valor menor que 0,05, utilizando o método Backward.

A pesquisa foi aprovada pelo Comitê de Ética em Pesquisa da UCB, sob parecer no 3.061.534, atendendo às exigências da Resolução no 466/12 do Conselho Nacional de Saúde, que dispõe sobre as normas e diretrizes regulamentadoras de pesquisas envolvendo seres humanos.

\section{RESULTADOS}

Os participantes da pesquisa foram, em maior parte, do sexo feminino, correspondendo a $61,5 \%$ da amostra, concentrando-se na faixa etária de 80 a 84 anos, 48,4\%, tendo a maioria autodeclarada branca, 68,1\%. Os solteiros representaram 43,1\% da amostra, possuindo, em sua maioria, 59,7\%, 4 ou mais filhos. A maior parte não trabalha e recebe aposentadoria (Tabela 1). 
Tabela 1. Variáveis sociodemográficas de pessoas idosas longevas atendidas no ambulatório do HUCB, 2016.

\begin{tabular}{|c|c|}
\hline Variáveis & Frequência \\
\hline \multicolumn{2}{|l|}{ Idade (em anos) } \\
\hline 78 a 79 & $14(15,4 \%)$ \\
\hline 80 a 84 & $44(48,3 \%)$ \\
\hline$>85$ & $32(35,2 \%)$ \\
\hline Não informado & $1(1,1 \%)$ \\
\hline \multicolumn{2}{|l|}{ Sexo } \\
\hline Masculino & $34(37,4 \%)$ \\
\hline Feminino & $56(61,5 \%)$ \\
\hline Não informado & $1(1,1 \%)$ \\
\hline \multicolumn{2}{|l|}{ Cor } \\
\hline Branco & $62(68,1 \%)$ \\
\hline Preto & $2(2,2 \%)$ \\
\hline Mulato & $23(25,3 \%)$ \\
\hline Indígena & $1(1,1 \%)$ \\
\hline Amarelo & $2(2,2 \%)$ \\
\hline Não informado & $1(1,1 \%)$ \\
\hline \multicolumn{2}{|l|}{ Trabalha } \\
\hline Sim & $12(13,2 \%)$ \\
\hline Não & $78(85,7 \%)$ \\
\hline Não informado & $1(1,1 \%)$ \\
\hline \multicolumn{2}{|l|}{ Aposentado } \\
\hline Sim & $75(82,4 \%)$ \\
\hline Não & $14(15,4 \%)$ \\
\hline Não informado & $2(2,2 \%)$ \\
\hline
\end{tabular}

As doenças crônicas mais relatadas foram hipertensão arterial, 80,5\%, osteoporose, 36,3\%, e diabetes, presente em $28,9 \%$ da amostra.

Em relação aos eventos estressores vivenciados nos últimos cinco anos, mais da metade relatou morte de ente querido (61\%). Outros eventos frequentes foram perda de atividade ou amizade que gostava muito (48\%), doença em ente querido (47\%), doença na própria pessoa $(38 \%)$, eventos ocorridos na descendência (34\%) e perda de poder aquisitivo $(33 \%)$. Os eventos menos relatados foram violência a própria pessoa idosa (27\%), conflitos familiares (21\%) e cuidados prestados a algum familiar (3\%). Os percentuais dos eventos estressantes estão representados na Figura 1.
A média dos valores das citocinas foi de $6,12 \mathrm{pg} /$ mLpara IFN $\gamma, 3,32 \mathrm{pg} / \mathrm{mL}$ para TNF, 3,54 pg/mL para IL-10, 12,27 pg/mL para IL-6, 1,70 pg/mL para IL-4 e 9,10 $\mathrm{pg} / \mathrm{mL}$ para IL-2.

Foi verificada a influência do conjunto de variáveis: sexo, faixa etária, anos de estudo, estado conjugal, cor, número de filhos, trabalho, aposentadoria, suspeição de depressão pela GDS, arranjo familiar e biomarcadores inflamatórios na presença de eventos estressores. Essa associação foi feita conforme o número de eventos assim divididos: 0 a 2; 3 e 4; 5 e mais. Nela o número de eventos estressores esteve diretamente relacionado a maiores chances de depressão e ao fato dessas pessoas idosas longevas não estarem trabalhando (Tabela 2). 
Em seguida, os dados com valores de $p$ menor que 0,20 foram submetidos à regressão linear, tendo como variável dependente o número de eventos estressores acrescentados todos os biomarcadores inflamatórios. A depressão foi a única variável significativa nessa análise de regressão linear, evidenciando que, quando mais eventos estressores, maior a pontuação no GDS.
Em uma segunda rodada, novamente foram retirados os dados com valores de $p$ menores que 0,20 reservando-se apenas as variáveis correspondentes aos biomarcadores inflamatórios, até que se chegasse às variáveis mais significativas, tendo todas valor de $p$ menor que 0,05 . Os dados obtidos estão representados na Tabela 3 .

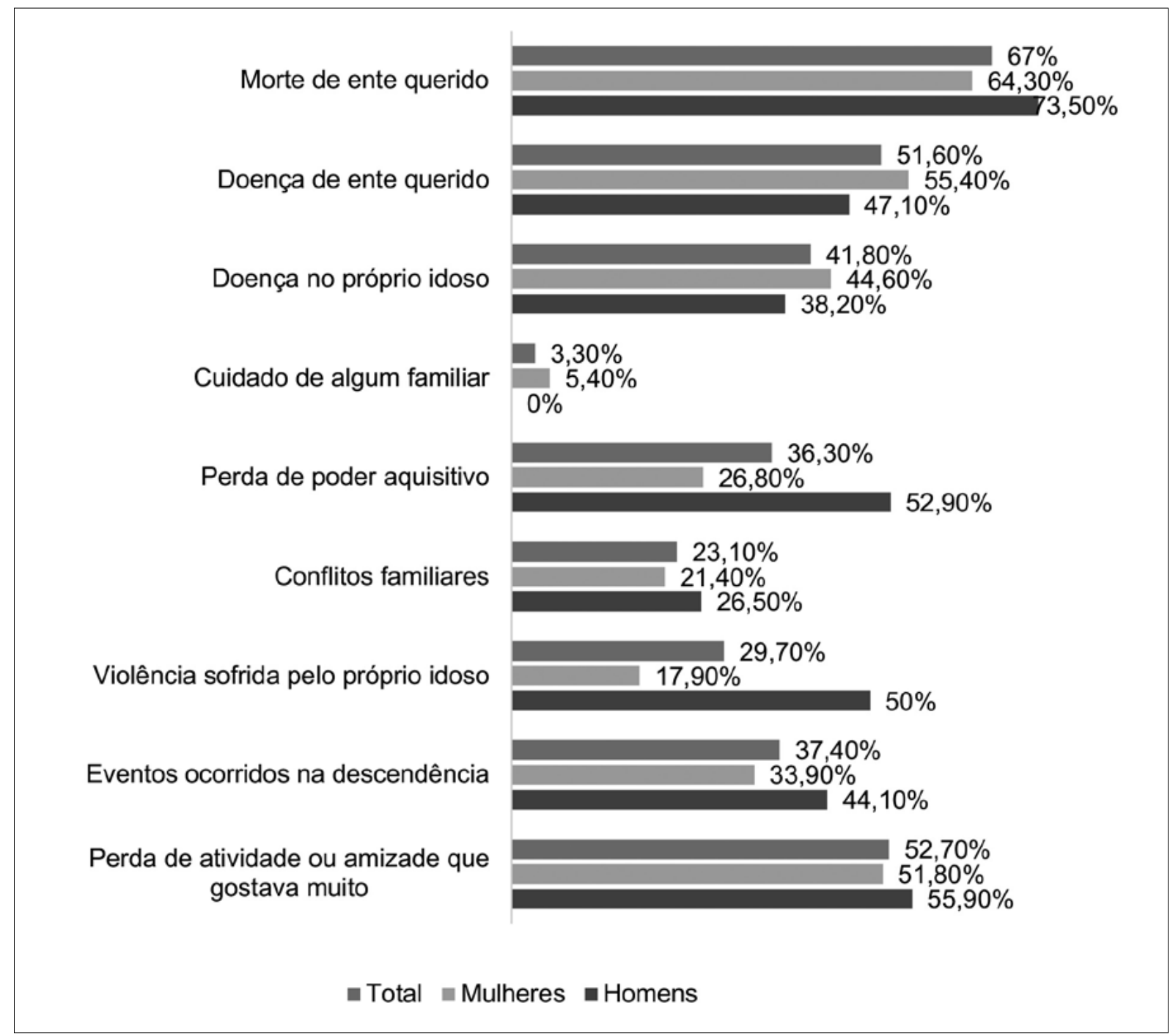

Figura 1. Número de eventos estressores apresentados por pessoas idosas longevos atendidos no ambulatório do HUCB, 2016. 
Tabela 2. Análise da relação entre o número de eventos estressores de vida e Biomarcadores inflamatórios e variáveis sociodemográficas em pessoas idosas longevas atendidas no ambulatório do HUCB, 2016.

\begin{tabular}{|c|c|c|c|c|c|}
\hline Variável & $\begin{array}{l}0 \text { a } 2 \text { eventos } \\
\text { estressores de vida }\end{array}$ & $\begin{array}{l}3 \text { e } 4 \text { eventos } \\
\text { estressores de vida }\end{array}$ & $\begin{array}{l}5 \text { e mais eventos } \\
\text { estressores de vida }\end{array}$ & Total & $p$-valor \\
\hline Total & 9 & 32 & 31 & 91 & \\
\hline IFN- $\gamma$ & & & & & $0,700^{\mathrm{KW}}$ \\
\hline Mediana (IQR) & $5,7(4,8-6,5)$ & $5,9(5,4-6,6)$ & $5,9(5,5-6,4)$ & $5,9(5,4-6,5)$ & \\
\hline $\mathrm{TNF}-\alpha$ & & & & & $0,680^{\mathrm{KW}}$ \\
\hline Mediana (IQR) & $2(1-3,1)$ & $2(1,3-2,5)$ & $1,8(1,1-2,4)$ & $1,9(1,3-2,5)$ & \\
\hline IL-10 & & & & & $0,572^{\mathrm{KW}}$ \\
\hline Mediana (IQR) & $3,2(3-3,3)$ & $3,7(3-3,9)$ & $3,6(2,9-3,8)$ & $3,6(2,9-4)$ & \\
\hline IL-6 & & & & & $0,933^{\mathrm{KW}}$ \\
\hline Mediana (IQR) & $5,2(3,8-5,6)$ & $4,8(3,4-8,7)$ & $4,6(3,1-7,9)$ & $4,8(3,2-7,2)$ & \\
\hline IL-4 & & & & & $0,307^{\mathrm{KW}}$ \\
\hline Mediana (IQR) & $1,9(1,9-2,7)$ & $1,6(1,3-2,1)$ & $1,5(1,2-2)$ & $1,6(1,3-2,1)$ & \\
\hline IL-2 & & & & & $0,699^{\mathrm{KW}}$ \\
\hline Mediana (IQR) & $8,9(8,8-9,1)$ & $9,2(8,6-9,7)$ & $8,9(8,6-9,5)$ & $8,9(8,6-9,4)$ & \\
\hline Sexo & & & & & $0,978^{\mathrm{Qq}}$ \\
\hline Masculino & $4(44,4 \%)$ & $13(40,6 \%)$ & $13(41,9 \%)$ & $30(41,7 \%)$ & \\
\hline Feminino & $5(55,6 \%)$ & $19(59,4 \%)$ & $18(58,1 \%)$ & $42(58,3 \%)$ & \\
\hline Faixa etária (em anos) & & & & & $0,555^{\mathrm{TF}}$ \\
\hline 78 a 79 & $1(11,1 \%)$ & $4(12,5 \%)$ & $6(19,4 \%)$ & $11(15,3 \%)$ & \\
\hline 80 a 84 & $3(33,3 \%)$ & $16(50,0 \%)$ & $17(54,8 \%)$ & $36(50,0 \%)$ & \\
\hline 85 e mais & $5(55,6 \%)$ & $12(37,5 \%)$ & $8(25,8 \%)$ & $25(34,7 \%)$ & \\
\hline Anos de estudo & & & & & $0,944^{\mathrm{TF}}$ \\
\hline Nenhum & $1(11,1 \%)$ & $7(21,9 \%)$ & $8(25,8 \%)$ & $16(22,2 \%)$ & \\
\hline 1 a 4 & $4(44,4 \%)$ & $14(43,8 \%)$ & $11(35,5 \%)$ & $29(40,3 \%)$ & \\
\hline 5 a 8 & $2(22,2 \%)$ & $6(18,8 \%)$ & $8(25,8 \%)$ & $16(22,2 \%)$ & \\
\hline 9 e mais & $2(22,2 \%)$ & $5(15,6 \%)$ & $4(12,9 \%)$ & $11(15,3 \%)$ & \\
\hline Estado civil & & & & & $0,074^{\mathrm{TF}}$ \\
\hline Casado & $1(11,1 \%)$ & $1(3,1 \%)$ & $4(12,9 \%)$ & $6(8,3 \%)$ & \\
\hline Solteiro & $1(11,1 \%)$ & $17(53,1 \%)$ & $13(41,9 \%)$ & $31(43,1 \%)$ & \\
\hline Divorciado & $1(11,1 \%)$ & $1(3,1 \%)$ & $5(16,1 \%)$ & $7(9,7 \%)$ & \\
\hline Viúvo & $6(66,7 \%)$ & $13(40,6 \%)$ & $9(29,0 \%)$ & $28(38,9 \%)$ & \\
\hline Número de filhos & & & & & $0,423^{\mathrm{TF}}$ \\
\hline 0 a 1 & $2(22,2 \%)$ & $4(12,5 \%)$ & $3(9,7 \%)$ & $9(12,5 \%)$ & \\
\hline 2 a 3 & $4(44,4 \%)$ & $9(28,1 \%)$ & $7(22,6 \%)$ & $20(27,8 \%)$ & \\
\hline 4 e mais & $3(33,3 \%)$ & $19(59,4 \%)$ & $21(67,7 \%)$ & $43(59,7 \%)$ & \\
\hline Trabalho & & & & & $0,022^{\mathrm{TF}}$ \\
\hline Sim & $4(44,4 \%)$ & $4(12,5 \%)$ & $2(6,5 \%)$ & $10(13,9 \%)$ & \\
\hline Não & $5(55,6 \%)$ & $28(87,5 \%)$ & $29(93,5 \%)$ & $62(86,1 \%)$ & \\
\hline Aposentadoria & & & & & $0,083^{\mathrm{TF}}$ \\
\hline Sim & $6(66,7 \%)$ & $30(93,8 \%)$ & $27(90,0 \%)$ & $63(88,7 \%)$ & \\
\hline Não & $3(33,3 \%)$ & $2(6,2 \%)$ & $3(10,0 \%)$ & $8(11,3 \%)$ & \\
\hline
\end{tabular}


Continuação da Tabela 3

\begin{tabular}{|c|c|c|c|c|c|}
\hline Variável & $\begin{array}{l}0 \text { a } 2 \text { eventos } \\
\text { estressores de vida }\end{array}$ & $\begin{array}{l}3 \text { e } 4 \text { eventos } \\
\text { estressores de vida }\end{array}$ & $\begin{array}{l}5 \text { e mais eventos } \\
\text { estressores de vida }\end{array}$ & Total & $p$-valor \\
\hline Depressão & & & & & $0,013^{\mathrm{Qq}}$ \\
\hline Sim & $0(0)$ & $5(16,7 \%)$ & $13(41,9 \%)$ & $18(25,7 \%)$ & \\
\hline Não & $9(100 \%)$ & $25(83,3 \%)$ & $18(58,1 \%)$ & $52(74,3 \%)$ & \\
\hline Sozinho & & & & & $0,964^{\mathrm{Qq}}$ \\
\hline Sim & $3(33,3 \%)$ & $10(31,2 \%)$ & $9(29,0 \%)$ & $22(30,6 \%)$ & \\
\hline Não & $6(66,7 \%)$ & $22(68,8 \%)$ & $22(71,0 \%)$ & $50(69,4 \%)$ & \\
\hline Cônjuge & & & & & $0,332^{\mathrm{Qq}}$ \\
\hline Sim & $2(22,2 \%)$ & $16(50,0 \%)$ & $14(45,2 \%)$ & $32(44,4 \%)$ & \\
\hline Não & $7(77,8 \%)$ & $16(50,0 \%)$ & $17(54,8 \%)$ & $40(55,6 \%)$ & \\
\hline Filhos & & & & & $0,574^{\mathrm{TF}}$ \\
\hline Sim & $6(66,7 \%)$ & $15(46,9 \%)$ & $17(54,8 \%)$ & $38(52,8 \%)$ & \\
\hline Não & $3(33,3 \%)$ & $17(53,1 \%)$ & $14(45,2 \%)$ & $34(47,2 \%)$ & \\
\hline Netos & & & & & $0,561^{\mathrm{Qq}}$ \\
\hline Sim & $4(44,4 \%)$ & $10(31,2 \%)$ & $8(25,8 \%)$ & $22(30,6 \%)$ & \\
\hline Não & $5(55,6 \%)$ & $22(68,8 \%)$ & $23(74,2 \%)$ & $50(69,4 \%)$ & \\
\hline Bisnetos & & & & & $0,184^{\mathrm{TF}}$ \\
\hline Sim & $1(11,1 \%)$ & $2(6,2 \%)$ & $0(0,0)$ & $3(4,2 \%)$ & \\
\hline Não & $8(88,9 \%)$ & $30(93,8 \%)$ & $31(100,0 \%)$ & $69(95,8 \%)$ & \\
\hline Outro parente & & & & & $0,626^{\mathrm{TF}}$ \\
\hline Sim & $0(0,0)$ & $1(3,1 \%)$ & $3(9,7 \%)$ & $4(5,6 \%)$ & \\
\hline Não & $9(100,0 \%)$ & $31(96,9 \%)$ & $28(90,3 \%)$ & $68(94,4 \%)$ & \\
\hline Amigo & & & & & $0,724^{\mathrm{TF}}$ \\
\hline Sim & $1(11,1 \%)$ & $2(6,2 \%)$ & $3(9,7 \%)$ & $6(8,3 \%)$ & \\
\hline Não & $8(88,9 \%)$ & $30(93,8 \%)$ & $28(90,3 \%)$ & $66(91,7 \%)$ & \\
\hline
\end{tabular}

IQR: Intervalo interquartil; KW: Kruskal-wallis; Qq: Qui-quadrado; TF: Teste de Fisher.

Tabela 3. Fatores associados ao número de eventos estressores entre pessoas idosas longevas no Distrito Federal.

\begin{tabular}{lll}
\hline Variáveis & Coeficiente (IC 95\%) & $p$-valor \\
\hline IFN & $0,34(-0,21-0,89)$ & 0,224 \\
TNF & $-0,02(-0,06-0,02)$ & 0,406 \\
IL-10 & $0,16(-0,49-0,8)$ & 0,624 \\
IL-6 & $0,03(-0,05-0,12)$ & 0,428 \\
IL-4 & $-0,66(-1,3--0,01)$ & $\mathbf{0 , 0 4 6}$ \\
IL-2 & $0(-0,72-0,72)$ & 1 \\
\hline Estado civil* & & \\
Solteiro & $-0,51(-2,06-1,03)$ & 0,508 \\
Divorciado & $-0,23(-2,08-1,62)$ & 0,804 \\
Viúvo & $-1,73(-3,36--0,11)$ & $\mathbf{0 , 0 3 7}$ \\
\hline Depressão & & $\mathbf{0 , 0 0 5}$ \\
Sim vs Não & $1,35(0,42-2,29)$ & \\
\hline
\end{tabular}

* Referência para estado civil: ser casado; IC: Intervalo de Confiança. 
Ao final, as variáveis que se demonstraram estatisticamente significativas foram a IL-4, a viuvez e a depressão. Assim, quanto mais eventos estressores foram relatados, menor a contagem de IL-4. Em relação à viuvez os dados mostraram que quem é viúvo tem 1,63\% menos eventos estressores em comparação a quem é casado. Os dados ainda mostraram que quem apresentou rastreio positivo para depressão (pontuação GDS $\geq 5$ ) tem $1,17 \%$ mais eventos estressores quando comparado a quem não tem depressão.

\section{DISCUSSÃO}

O objetivo principal do presente estudo foi avaliar a relação entre as citocinas e eventos estressores em pessoas idosas longevas, onde se observou relação significativa entre maior número de eventos e menores concentrações de IL-4.

Nossos resultados também demonstraram que existe uma forte associação entre o número de eventos estressores e presença de sintomas depressivos, observada em $25,7 \%$ da amostra. Fato semelhante também observado em estudo realizado com 385 pessoas idosas chinesas residentes na comunidade, onde foi observado que o aumento do número de eventos estressores de vida e menores níveis de resiliência foram significativamente associados a níveis mais altos de sintomatologia depressiva ${ }^{22}$.

O estresse pode se tornar ainda fator agravante para alterações imunológicas, em especial na velhice, já caracterizada também pela imunossenescência. Dentre os mediadores inflamatórios analisados, a IL-4 apresentou associação inversamente proporcional aos eventos estressores.

Além das funções imunológicas anti-inflamatórias já conhecidas, a IL-4 mostra indícios de relação com comportamentos depressivos, como observado em pesquisa de Wachholz et $\mathrm{al}^{23}$. Esses autores, usando interferon- $\alpha$ em ratos, observaram que uma menor responsividade à IL-4 da micróglia estava especificamente relacionada ao desenvolvimento de comportamento depressivo. Assim, a IL-4 parece estar relacionada à regulação do comportamento depressivo em uma condição não tratada. Porém, esse efeito foi evidente apenas em combinação com uma disposição genética adicional que parece estar ausente em determinada cepa de camundongos ${ }^{23}$.

Já em estudo realizado por Lee et al. ${ }^{24} \mathrm{em}$ camundongos submetidos a estresse por imobilização, observou-se diminuição significativa na secreção de IL-4 no tronco cerebral, em relação ao grupo controle, e relação inversa entre a ativação dos componentes neuroendócrinos e neuronais primários da resposta ao estresse e concentração de IL-4.

Essa interleucina foi descoberta pela primeira vez como fator secretado por células $\mathrm{T}$, promovendo o aumento da proliferação das células B estimuladas por anti-IgM em $1982^{25}$. É agora reconhecida como reguladora de uma ampla variedade de funções nas células imunes como linfócitos Th2, basófilos, eosinófilos e mastócitos, tem receptores expressos em muitos tipos de células, podendo estimular a proliferação e diferenciação celular, regeneração de tecidos e funções neurológicas ${ }^{25,26}$.

A IL-4 é uma citocina reguladora por excelência, desempenhando um vasto papel na função imune, com funções anti-inflamatórias cada vez mais reconhecidas ${ }^{26}$. No momento da sua descoberta, pouco se imaginava quão ampla seria sua funcionalidade, nem se previa a possibilidade de que bloquear seu receptor provasse ser uma estratégia valiosa, como foi demonstrado com a eficácia da criação de um medicamento para asma moderada a grave e em dermatite atópica ${ }^{26}$.

A ativação imunológica inflamatória tem sido frequentemente associada ao desenvolvimento da depressão maior e a micróglia (células imunológicas cerebrais) pode servir como uma interface importante na comunicação desse sistema com o cérebro. A IL4, principal citocina do tipo Th2, pode atuar como protetora contra a depressão devido à capacidade de regular negativamente processos inflamatórios e inibir a atividade transportadora de serotonina ${ }^{24}$.

Em relação ao estado civil, os viúvos apresentaram menor número de eventos estressores quando comparado aos casados. Esse fato pode ser confrontado com resultado obtido por estudo de Trevisan et $\mathrm{al}^{27}$ que mostrou que, enquanto homens 
idosos podem sofrer consequências negativas após a morte da companheira, as mulheres idosas viúvas aparentaram ficar mais saudáveis.

Apesar deste estudo não ter revelado em seus resultados o estado civil dos participantes de acordo com sexo masculino ou feminino, a maior parte da amostra foi composta por mulheres, que em decorrência de funções sociais tradicionalmente típicas como cuidadoras dos cônjuges e descendentes assumindo tarefas domésticas com peso de "obrigação", tendem a sofrer mais fadiga e ansiedade ${ }^{27}$.

Vários estudos relacionam estresse emocional e alterações no padrão imunológico, onde se destacam como mais estudadas as citocinas IL-1, IL-6 e $\mathrm{TNF}-\alpha^{28}$, entre os quais pode-se citar uma metanálise realizada por realizada por Black e Miller ${ }^{29}$, onde observou-se níveis elevados de IL-1 $\beta$ e IL-6 em amostras cerebrais e sanguíneas após a morte de pessoas com tendência suicida em comparação com controles saudáveis e sem suicídio e níveis de IL-8 menores no líquido cefalorraquidiano em indivíduos que exibem comportamento suicida.

Entretanto, o número limitado de publicações de estudos relacionando outras citocinas como a IL-4 às alterações neurológicas em resposta ao estresse sugere ampla lacuna de conhecimento. Essa situação coloca em local de destaque pesquisas que investigam a temática em populações específicas como esta, com enfoque para populações idosas.

O inflammaging possui grande impacto para a pessoa idosa, culminando em níveis basais de citocinas pró-inflamatórias aumentadas ${ }^{10-12,15}$, com alterações inda mais intensas com o avançar da idade, em especial na faixa etária estudada, fato observado nos níveis elevados de dosagem dessas citocinas na amostra.

O desequilíbrio na produção de citocinas pode ainda ser exacerbado pela presença de eventos com potencial estressor, que podem atuar como potencializadores da atividade inflamatória ${ }^{9}$. No presente estudo, foi observada uma relação entre menores concentrações de IL-4 e maior número de eventos estressores.
Dada a alta frequência de mulheres na amostra, o número de eventos apresentados, a relação desse número com o estado conjugal e o relato de violência evidenciam a necessidade de políticas públicas que tenham como foco a proteção dos direitos das pessoas idosas, em especial da mulher.

Além disso, um olhar mais cuidadoso dos profissionais de saúde na avaliação multidimensional da pessoa idosa, de forma que se obtenham subsídios para a implementação de programas e intervenções específicas que possam amenizar a percepção dos eventos estressores vivenciados, identificandoos precocemente e fortalecendo mecanismos de enfrentamento ${ }^{22,28}$ para uma velhice mais ativa $\mathrm{e}$, inclusive, com menores danos decorrentes da imunossenescência.

O presente estudo apresenta algumas limitações, como o tamanho da amostra. Acreditamos que isso se deve ao perfil dos idosos desse estudo (longevos) e à dependência de terceiros para participarem das coletas de dados, onde muito idosos deixaram de participar das etapas. Em relação ao estágio de validação do Inventário de Eventos Estressores de Vida, não foram encontradas publicações para a população brasileira, apesar de já bastante utilizado em pesquisas nessa população. Além disso, não foi avaliado o nível de estresse atribuído a cada evento, somente a presença ou não da ocorrência de cada evento estressor. Não foram encontrados na literatura estudos parecidos em seres humanos relacionando especificamente a IL-4 a eventos estressores, por esse motivo consideramos que o presente estudo ganha grande relevância por se tratar de temática ainda pouco estudada e pela necessidade de apresentar dados de pessoas idosas com idade média superior a oitenta anos no Brasil.

\section{CONCLUSÃO}

A realização deste estudo permitiu verificar a relação entre a presença de eventos estressores e biomarcadores inflamatórios, onde se destacou a diminuição da IL-4, que possui função antiinflamatória, com o aumento do número de eventos. Assim, nota-se a relevância na continuidade de 
estudos que aprofundem a relação entre essa citocina e os aspectos psicológicos, especialmente em pessoas idosas longevas.

Ao conhecer a frequência dos eventos foi possível verificar relação com maior frequência de sintomas depressivos e menor risco a esses eventos entre as pessoas idosas viúvas quando comparadas com as casadas.

Os resultados trazem contribuições importantes para o desenvolvimento de políticas públicas para melhoria da qualidade de vida da população idosa, como a evidência da importância da investigação dos eventos estressores nessa população.

\section{REFERÊNCIAS}

1. Ansari S. Stress of aged elderly: a review.

Indian J Health Wellbeing. 2019;10(4):162-5.

Disponível em: https://www.proquest.com/

docview $/ 2307090317$ ?pqorigsite $=$ gscholar\&fromo penview $=$ true

2. Fontes AP, Lemos LC, Neri AL. Experiência de eventos de vida estressantes na velhice e de riscos e adversidades na infância e adolescência. In: Neri AL, Borim FS, Assumpção D, editors. Octogenários em Campinas: dados do fibra $80+$. Campinas: Alinea; 2019. p. 223-38.

3. Neri AL, Yassuda MS. Velhice bem-sucedida: aspectos afetivos e cognitivos. Campinas: Papirus; 2004.

4. Surachman A, Almeida DM. Stress and Coping Theory Across the Adult Lifespam. Dev Psychol. 2018:1-9. Disponível em: https://doi.org/10.1093/ acrefore/9780190236557.013.341

5. Aldwin CM. Stress, coping, and development: an integrative perspective. $2^{\mathrm{a}}$ ed. New York: Guilford Press; 2009.

6. Baltes PB. Theoretical propositions of life-spam developmental psychology: on the dynamics between growth and decline. Dev Psychol. 1987;23(5):61126. Disponível em: https://doi.org/10.1037/00121649.23.5.611

7. Fortes ACG, Neri AL. Eventos de vida e envelhecimento humano. In: Neri AL, Yassuda MS. Velhice bem-sucedida: aspectos afetivos e cognitivos. $3^{\mathrm{a}}$ ed. Campinas: Papirus; 2008. p. 51-70.
Compreende-se, portanto, a necessidade de acolhimento e acompanhamento por parte dos profissionais de saúde na prática clínica que leve em consideração os eventos com potencial estressor para criação de um arcabouço que os auxilie a conviver ou mesmo superar, favorecendo a resiliência.

O investimento público em estratégias de enfrentamento e criação de redes de apoio como ação de promoção à saúde pode otimizar os recursos em saúde pública, pelo potencial de amenizar os danos decorrentes dos efeitos negativos relacionados à imunossenescência.

Editado por: Maria Luiza Diniz de Sousa Lopes

8. Dantzer R, Cohen S, Russo S, Dinan TG.

Resilience and immunity. Brain Behav Immun Health. 2018;74:28-42. Disponível em: https://doi. org/10.1016/j.bbi.2018.08.010

9. Fali T, Vallet H, Sauce D. Impact of stress on aged immune system compartments: Overview from fundamental to clinical. Exp Gerontol. 2018;5:1926. Disponível em: https://doi.org/10.1016/j. exger.2018.02.007

10. Pawelec G. Age and immunity: What is immunosenescence? Exp Gerontol. 2018;105:49. Disponível em: https://doi.org/10.1016/j. exger.2017.10.024

11. Esquenazi DA. Imunossenescência: as alterações do sistema imunológico provocadas pelo envelhecimento. Rev Hosp Univ Pedro Ernesto. 2008;7(1): 38-45. Disponível em: https://www.arca.fiocruz.br/handle/ icict $/ 28948$

12. Flynn MG, Markofski MM, Carrillo AE. Elevated Inflammatory Status and Increased Risk of Chronic Disease in Chronological Aging: Inflamm-aging or Inflamm-inactivity? Aging Dis. 2019;10(1):14756. Disponível em: https://doi.org/10.14336/ AD.2018.0326

13. Milan-Mattos JC, Anibal FF, Perseguini NM, Minatel V, Santos PR, Castro CA, et al. Effects of natural aging and gender on pro-inflammatory markers. Braz J Med Biol Res. 2019;52(9):1-9. Disponível em: http:// dx.doi.org/10.1590/1414-431x20198392 
14. Fulop T, Larbi A, Witkowski JM. Human Inflammaging. Gerontology. 2019;65(5):495-504. Disponível em: https://doi.org/10.1159/000497375

15. Minciullo PL, Catalano A, Mandraffino G, Casciaro M. Inflammaging and Anti-Inflammaging: The Role of Cytokines in Extreme Longevity. Arch Immunol Ther Exp. 2015;(64):111-26. Disponível em: https:// doi.org/10.1007/s00005-015-0377-3

16. Fulop T, Larbi A, Dupuis G, Page AL, Frost EH, Cohen AA, et al. Immunosenescence and InflammAging As Two Sides of the Same Coin: Friends or Foes?. Front Immunol. 2018. Disponível em: https:// doi.org/10.3389/fimmu.2017.01960

17. Brucki MD, Nitrini R, Caramelli P, Bertolucci HF, Okamoto IH. Sugestões para o uso do mini-exame do estado mental no Brasil. Arq Neuropsiquiatr. 2003;61(3B):777-81. Disponível em: https://doi. org/10.1590/S0004-282X2003000500014

18. Aldwin CM. The Elders Life Stress Inventory: Egocentric and nonegocentric stress. In: Stephens MP, Crowther JH, Hobfoll SE, Tennenbaum DL, Stephens MP, Crowther JH, et al. Stress and coping in later-life families. Washington, DC: Hemisphere Publishing Corp; 1990. p. 49-69.

19. Yesavage JA, Brink TL, Rose TL, Lum O, Huang $\mathrm{V}$, Adey M, et al. Development and validation of a geriatric depression screening scale: a preliminary report. J Psychiatr Res. 1983;17(1):37-49. Disponível em: https://doi.org/10.1016/0022-3956(82)90033-4

20. Almeida OO, Almeida SA. Confiabilidade da versão brasileira da Escala de Depressão em Geriatria (GDS) versão reduzida.

Arq Neuropsiquiatr. 1999;57(2B):421-6.

Disponível em: http://dx.doi.org/10.1590/S0004282X1999000300013

21. Paradela EM, Lourenço RA, Veras RP. Validação da escala de depressão geriátrica em um ambulatório geral. Rev Saúde Pública. 2005;39(6):918-23. Disponível em: http://dx.doi.org/10.1590/S003489102005000600008
22. Lim ML, Gwee DL, Nyunt MS, Kumar R, Ng TP. Resilience, stressful life events, and depressive symptomatology among older Chinese adults. Aging Ment Health. 2015;19(11):1005-14. Disponível em: https://doi.org/10.1080/13607863.2014.995591

23. Wachholz S, Mengert L, Plümper J, Sommer R, Juckel G, Friebe A. Interleukin-4 is a participant in the regulation of depressive-like behavior. Brain Res Bull. 2017;326:165-72. Disponível em: https://doi. org/10.1016/j.bbr.2017.03.020

24. Lee H, Park HJ, Starkweather A, An K, Shim I. Decreased Interleukin-4 Release from the Neurons of the Locus Coeruleus in Response to Immobilization Stress. Mediat Inflamm. 2016. Disponível em: https://doi.org/10.1155/2016/3501905

25. Paul EW. History of interleukin-4. Cytokine. 2015;75(1):3-7. Disponível em: https://doi. org/10.1016/j.cyto.2015.01.038

26. Howard M, Farrar J, Hilfiker M, Johnson B, Takatsu $\mathrm{K}$, Hamaoka $\mathrm{T}$, et al. Identification of a $\mathrm{T}$ cell-derived $\mathrm{b}$ cell growth factor distinct from interleukin 2. J Exp Med. 1982;155(3):914-23. Disponível em: https://doi. org/10.1084/jem.155.3.914

27. Trevisan C, Grande G, Vetrano DL, Maggi S, Sergi G, Welmer AK, et al. Gender Differences in the Relationship Between Marital Status and the Development of Frailty: a Swedish Longitudinal Population-Based Study. J Womens Health. 2020;29(7):1-10. Disponível em: https://doi. org/10.1089/jwh.2019.8095

28. Slavich GM. Psychoneuroimmunology of Stress and Mental Health. In: Harkness LK, Hayden EP. The Oxford Handbook of Stress and Mental Health. New York: Oxford University Press; 2020. p. 519-46.

29. Black C, Miller BJ. Meta-analysis of cytokines and chemokines in suicidality: Distinguishing suicidal versus nonsuicidal patients. Biol Psychiatry. 2015;78(1):28-37. Disponível em: https://doi. org/10.1016/j.biopsych.2014.10.014 